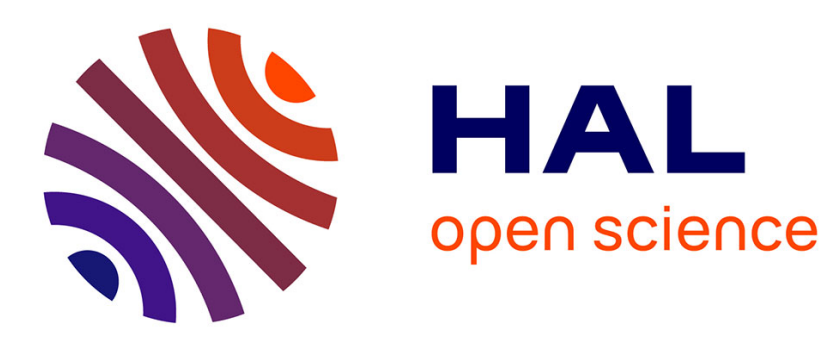

\title{
Économie morale du pèlerinage et société civile en Iran. Les voyages religieux, commerciaux et touristiques à Damas
}

Fariba Adelkhah

\section{- To cite this version:}

Fariba Adelkhah. Économie morale du pèlerinage et société civile en Iran. Les voyages religieux, commerciaux et touristiques à Damas. Politix, 2007, 77, pp.39 - 54. 10.3917/pox.077.0039 . hal03384654

\section{HAL Id: hal-03384654 \\ https://hal.science/hal-03384654}

Submitted on 19 Oct 2021

HAL is a multi-disciplinary open access archive for the deposit and dissemination of scientific research documents, whether they are published or not. The documents may come from teaching and research institutions in France or abroad, or from public or private research centers.
L'archive ouverte pluridisciplinaire HAL, est destinée au dépôt et à la diffusion de documents scientifiques de niveau recherche, publiés ou non, émanant des établissements d'enseignement et de recherche français ou étrangers, des laboratoires publics ou privés. 


\title{
Économie morale du pèlerinage et société civile en Iran : les voyages religieux, commerciaux et touristiques à Damas
}

Fariba AdELKHAH

\begin{abstract}
Résumé - Le pèlerinage est une pratique religieuse centrale dans l'islam. Mais sous ce constat banal se cache une grande complexité sociale et historique. D'une part, le pèlerinage n'est pas un phénomène atemporel. II est situé dans le temps et bien sûr dans l'espace. D'autre part, il comporte des aspects extra-religieux, mondains ou profanes. Cette complexité fait du pèlerinage une pratique politique notable. L'observation participante d'un voyage de pèlerins téhéranais aux lieux saints chiites de Damas et Alep, en juillet 2003, permet de dégager le rôle que les femmes y exercent désormais, à la fois sur les plans de la religiosité et du commerce à la valise, et les conséquences sociales qui en découlent du point de vue des relations de genre, de l'autonomisation des rapports marchands eu égard au pouvoir politique et des processus d'individuation. L'expérience religieuse, tout en gardant sa transcendance et son irréductibilité, va de pair avec l'omniprésence du calcul économique rationnel : le pèlerinage est moins l'actualisation d'une communitas de croyants que la constitution d'un champ de pratiques et de luttes sociales, dans le double contexte de la nation et de la globalisation.
\end{abstract}




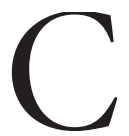

omme chacun sait le pèlerinage est une pratique religieuse centrale dans l'islam sans être spécifique à celui-ci. Mais sous ce constat banal se cache une grande complexité sociale et historique. D'une part, le pèlerinage n'est pas un phénomène atemporel. Il est situé dans le temps et bien sûr dans l'espace. D'autre part, il comporte des aspects extra religieux et mondains ou profanes ${ }^{1}$. Comme le disent Dale Eickelman et James Piscatori, ses motivations sont « inévitablement mêlées - une combinaison de raison sainte et de préoccupations sociales, économiques et politiques $»^{2}$. C'est cette complexité qui nous amène à étudier le pèlerinage dans sa polysémie sociale, et sous l'angle de sa contribution à la formation d'une société civile ou d'un espace public dans la République islamique d'Iran. Sa particularité provient notamment de son rapport simultané à l'économie morale et à la dimension transnationale du champ politique.

Pour démêler cet écheveau nous partirons de l'observation participante d'un pèlerinage d'un autocar de fidèles chiites iraniens sur les lieux saints de Syrie qui abritent notamment, à Damas et aux environs, les sépultures de Hazrat-e Roghiyeh - la fille de l'imam Hosssein, morte à l'âge de trois ans -, de Zeynab la sœur de l'imam Hossein -, de Ras-ol Hossein - la tête de l'imam Hossein -, et de la plupart des 72 compagnons de ce dernier tués lors de la bataille de Karbala, et, à Alep, la relique d'une goutte de sang de l'imam Hossein ${ }^{3}$. Traditionnellement les chiites iraniens se rendaient en pèlerinage, pour l'essentiel, à Mashhad, où repose le huitième imam Reza, à Karbala et Nadjaf, les grandes villes saintes du chiisme, en territoire irakien, et naturellement à La Mecque. Quelle que fût la dévotion dont Zeynab faisait l'objet, en particulier dans les représentations rituelles de Tazieh, Damas n'est entrée dans la géographie religieuse des chiites iraniens qu'en 1976, lorsque le sociologue Ali Shariati, figure emblématique de la gauche islamique disparue à Londres dans des conditions sujettes à polémique ${ }^{4}$, a été enterré, conformément à sa demande, aux côtés de la sœur de l'imam Hossein, qu'il vénérait pour son courage et son esprit de résistance. Mais il fallut attendre les années de la guerre contre l'Irak pour que la Syrie devienne une destination religieuse prisée. En effet les Iraniens qui voulaient se rendre à Nadjaf et Karbala en dépit du conflit, pour s’y

\footnotetext{
1. Outre les travaux classiques sur le pèlerinage par rapport auxquels notre article se situe en décroché pour reposer d'abord sur un travail de terrain et d'observation participante, nous voudrions signaler la publication de l'excellent livre collectif dirigé par S. Chiffoleau et A. Madoeuf: Les pèlerinages au Maghreb et au Moyen-Orient. Espaces publics, espaces du public, Beyrouth, Institut français du Proche-Orient, 2005.

2. Eickelman (D.), Piscatori (J.), eds, Muslim Travellers. Pilgrimage, migration, and the religious imagination, Berkeley, University of California Press, 1990, p. XIV.

3. Il faut en outre ajouter les sanctuaires dédiés à différentes personnalités chères au cœur des chiites, telles que des enfants et des compagnons du Prophète, des femmes de sa tribu, des savants. Pour une meilleure compréhension des lieux saints chiites en Syrie, cf. Mervin (S.), "Sayyida Zaynab, banlieue de Damas ou nouvelle ville sainte chiite », Cemoti, 22, 1996 et Ababsa (M.), "Significations territoriales et appropriations conflictuelles des mausolées chiites de Raqqa (Syrie) », in Chiffoleau (S.), Madoeuf (A.), dir., Les pèlerinages au Maghreb et au Moyen-Orient, op. cit.
}

4. Ali Shariati est mort d'une crise cardiaque. L'opposition a soupçonné la SAVAK de l'avoir assassiné. 
recueillir, pour visiter des parents ou pour y enterrer une dépouille selon l'usage ancestral, devaient, pour la majorité d'entre eux, emprunter ce détour. De même les derniers Irakiens d'origine iranienne qui ont fui le régime de Saddam Hussein sont sortis du pays par la Syrie. Les Moâved (littéralement les expulsés, c'est-à-dire les Irakiens d'origine iranienne que Saddam Hussein a contraints à l'exil par vagues successives à partir de la fin des années $1960^{5}$ ) ont d'ailleurs joué un rôle déterminant dans la découverte ou l'«invention » du pèlerinage à Zeynabieh, situé à sept kilomètres de Damas, et continuent d'en contrôler au moins la logistique hôtelière. Le voyage à Damas, dans le contexte du commerce informel lié à la situation de guerre, s'est également imposé, de pair avec d'autres déplacements, par exemple à Dubaï ou à Istanbul. Avec le retour à la paix en 1988, et la libéralisation du commerce extérieur, l'essor du commerce de valise et plus largement le développement de formes inédites de déplacement touristique qui s'en sont suivis, Damas a été visitée par un nombre croissant d'Iraniens et est aujourd'hui l'une de leurs destinations de prédilection, que desservent une noria d'autocars affrétés par des agences ou des particuliers et, depuis 2002, deux trains hebdomadaires. On estime à un million par an les touristes iraniens se rendant en Syrie, et à plusieurs centaines par jour le nombre des autocars qui franchissent la frontière ${ }^{6}$.

Nous avons quant à nous suivi ce pèlerinage du $1^{\mathrm{er}}$ au 14 juillet 2003, dans un autocar qu'avait affrété une agence et qu'une particulière avait contribué à remplir en servant d'intermédiaire. Dans les faits l'équipée était ainsi conduite par un binôme informel : d'une part, le guide de l'agence, de l'autre, la "dame » (Haj khanoum) qui tirait son ascendant de son rôle commercial d'intermédiaire et de sa qualité d'organisatrice de réunions religieuses dans son quartier. Les deux responsables étaient assistés du chauffeur et de ses deux aides, d'un caméraman vidéo, d'un cuisinier et d'un "garçon d'honneur » (eftekhâri) qui s'était mis au service quotidien du groupe en guise de paiement de son voyage, selon une pratique courante. Au nombre de 45 , soit sensiblement plus que ne l'aurait voulu la réglementation, les voyageurs s'étaient pour leur part acquitté d'une somme variable selon qu'ils étaient passés par l'agence ou par Haj khanoum: respectivement 130000 et 140000 touman ( 1 euro $=1010$ touman en 2003). Le jour du départ l'autocar a d'abord recueilli les passagers de l'agence avant de prendre ceux de Haj khanoum. Mais l'un des traits notables du voyage est que les deux groupes se sont vite fondus dans une même sociabilité.

5. Adelkhah (F.), «Transformation sociale et recomposition identitaire dans le Golfe : parfois malgré eux, toujours entre deux », Cemoti, 22, 1996.

6. M. Ababsa (art. cité, p. 113) donne le chiffre de 232985 pèlerins en 2002, en se fondant sur les statistiques syriennes. Il faudrait toutefois signaler que les pèlerins voyageant en bus n'ont pas de visa individuel mais bénéficient d'un visa collectif par autobus. En outre le nombre de voyageurs par bus, qui officiellement ne devrait pas dépasser les 40, atteint en réalité souvent les 50 passagers. 
L'autocar a franchi la frontière irano-turque au bout d'une quinzaine d'heures de route, s'est dirigé vers Erzurum, puis vers Malatya et Gaziantep, avant de franchir Alep et de parvenir enfin à Damas après quatre jours de route. Le retour s'est effectué grosso modo dans le même temps et selon le même itinéraire.

S'il ne fallait retenir qu'une caractéristique de cette équipée, ce serait son organisation et la capacité du groupe à s'y conformer de bon gré en dépit de son hétérogénéité. Ainsi, par exemple, de la préparation des repas. Elle était assurée par le cuisinier, comme il se doit. Mais rapidement chacun mit la main à la pâte tandis que l'un des voyageurs, un colonel à la retraite, nettoya systématiquement la nappe pour en avoir fait le vœu. En dépit de la fatigue une cinquantaine de personnes mangeaient de la sorte en une heure, une heure un quart. Derrière les règles de politesse en cours dans la société iranienne, l'habitude du vivre ensemble et la communion des pèlerins dans le but religieux du voyage intervenait une programmation quasi militaire de la part de l'agence. Ã aucun moment nous ne manquâmes de quoi que ce soit, si ce n'est d'eau au cours du dernier jour - mais il s'agissait sans doute d'une mesure de rétorsion de la part de l'aide chauffeur qui avait compris qu'il n'aurait pas de pourboire! Une organisatrice de pèlerinage, rencontrée à Damas, nous expliqua d'ailleurs qu'elle prévoyait les repas de façon très minutieuse et selon un ordre immuable: du pain, du raisin et du fromage, du beurre et de la confiture au petit-déjeuner; du riz et un plat en sauce chaque midi ; « un repas sec » (i.e. un sandwich avec de la viande grillée ou du thon, ou une galette d'herbes et de pommes de terre) le soir - et bien sûr thé et fruits à volonté toute la journée. Les soirs passés à l'hôtel permettaient en outre de servir du potage. La fiabilité de la logistique, singulièrement alimentaire, est une condition sine qua non de la paix des pèlerinages, et les agences ou les organisateurs individuels y jouent une bonne part de leur réputation.

De façon générale l'attente qu'aurait provoquée le retard ou le mauvais vouloir de certains voyageurs a été à peu près inexistante, ce qui est remarquable dans un groupe et a fortiori dans une société qui se caractérise, dit-on, par le non-respect systématique de toute règle et de tout horaire. Pace Gobineau! De là à voir dans l'autocar de pèlerins une communitas ${ }^{7}$ unie par la foi, il y a un pas, que nous ne franchirons pas tant cette dernière recouvrait des lignes de clivage et de conflit d'intérêts sur lesquels nous aurons à revenir. Néanmoins la pratique du pèlerinage atteste la vitalité et la viabilité d'une forme de civilité socialement et économiquement autonome dont il faudra se demander, le moment

\footnotetext{
7. Certes la notion de communitas telle que l'utilise V. Turner ne semble pas s'opposer à l'idée de processus social comme le souligne C. Decobert dans sa conclusion à Chiffoleau (S.), Madoeuf (A.), dir., Les pèlerinages au Maghreb et au Moyen-Orient, op. cit., p. 402. Mais l'usage courant que l'on a du terme renvoie plutôt à une communauté de corps unie par la foi et scellée une fois pour toutes et a tendance à occulter sa dimension processuelle.
} 
venu, si elle relève du concept de société civile. En revanche, qu'elle renvoie à une économie morale est une évidence et presque une tautologie. À travers le pèlerinage s'incarne une représentation éthique, religieuse et symbolique du monde que résume le déplacement initiatique, en réponse à un appel (talabidan) - en l'occurrence pour se recueillir sur la tombe de Hazrat-e Zeynab, qui se traduit par un sentiment d'accomplissement personnel et qui donne lieu à toute une série de pratiques sociales en matière de recueillement, de partage, de don, d'entraide, de distinction. Or, une telle économie morale est aussi bien politique, par le double rapport à la marchandise et à l'appartenance nationale qu'elle implique.

\section{Un rituel à dominante féminine}

Rien n'est moins «traditionnel » que ce pèlerinage à Damas. Outre le fait qu'il est, somme toute, de création récente, il signale l'une des grandes mutations de la société iranienne depuis une vingtaine d'années. Jadis le pèlerinage à La Mecque était une initiative quasi exclusive des hommes et même des notables, et celui de Mashhad avait une connotation familiale. Aujourd'hui ce sont aussi bien des jeunes que des femmes qui prennent la route pour s'aventurer à des milliers de kilomètres de chez eux, non seulement dans l'espace international mais aussi en dehors du cadre familial jadis constitutif de l'expédition pérégrine. En d'autres termes la pratique du pèlerinage s'est démocratisée, même si elle demeure un critère de distinction sociale et suppose un certain niveau de revenus en raison de son coût. Et de ce point de vue le pèlerinage en Syrie est particulièrement révélateur de cette transformation. Les femmes en sont la pierre angulaire, à la fois en tant que fidèles et en tant qu'organisatrices. L'initiative du départ se prend fréquemment dans le cadre des jalesseh, ces réunions religieuses féminines qui se sont multipliées depuis la Révolution ${ }^{8}$. Dans les autocars et dans les sanctuaires eux-mêmes le noir ou les fleurs des tchadors dominent. Cela peut s'expliquer assez trivialement par le fait que les femmes ont moins d'engagements professionnels, et donc plus de possibilités de partir une quinzaine de jours. Cependant cette interprétation est insatisfaisante - les femmes ont aussi plus de charges familiales et ménagères - et ne dit rien de l'essentiel, c'est-à-dire de la signification sociale du voyage lui-même. Par son intermédiaire les femmes s'affirment dans l'espace public, acquièrent un savoir faire spécifique, développent des formes de sociabilité propres, font irruption sur la scène internationale ou en tout cas régionale, changent les rapports sociaux au sein des familles et des quartiers, accumulent du capital ou des revenus autonomes par le biais du commerce de valise auquel elles se livrent à l’occasion du pèlerinage. 
Considérons par exemple le cas de Haj khanoum. Mariée à un épicier et servant à l'occasion dans la boutique, elle organise des réunions religieuses à son domicile. C'est dans l'assistance de ces dernières qu'elle recrute l'essentiel des pèlerines qui l'accompagnent à Damas, d'autres venant par le bouche à oreille. Lors du pèlerinage auquel nous avons participé Haj khanoum en était à son dixseptième voyage. Mais elle monte également des déplacements à l'intérieur de l'Iran, en particulier pour visiter les sources d'eau chaude, réputées être pleines de vertus thérapeutiques, de Sareine, dans la province d'Ardebil. Tantôt elle travaille en joint venture avec l'agence, et dans ce cas elle s'en remet à celle-ci pour tout ce qui a trait à la logistique, y compris l'obtention du visa syrien. Tantôt elle loue elle-même un autocar lorsqu'elle obtient suffisamment d'inscriptions, et elle prend alors en charge l'organisation matérielle du voyage. Comme on l'a vu, le pèlerinage que nous avons accompagné relevait du premier cas de figure. Néanmoins Haj khanoum exerçait une autorité certaine au sein de notre petite collectivité, forte de son expérience personnelle et de la vingtaine de pèlerines qu'elle avait amenées à l'agence, moyennant deux places gratuites pour ellemême. Aux dires de certaines des voyageuses, elle avait enjoint au chauffeur, lors d'un déplacement précédent, de rebrousser chemin sur une quarantaine de kilomètres pour prendre le petit-déjeuner sur les bords du lac de Van, conformément à ses habitudes et pour le plaisir des passagers. Auréolée de cet ascendant, elle entretenait avec le guide une rivalité feutrée, ne manquant pas de souligner que son mari aurait mieux fait s'il avait été là, et commandait sans beaucoup de retenue à l'équipage. Campant à l'arrière de l'autobus avec ses compagnes de voyage, elle s'était aménagée un semblant de couchette dans laquelle elle pouvait dormir, recouverte de son tchador, quand les autres voyageurs devaient somnoler recroquevillés sur leur siège. Elle s'activait au milieu de l'équipage - masculin - pour distribuer les repas sans gêne aucune. Mais bien entendu sa grande affaire, là où elle excellait beaucoup plus qu'en matière religieuse, faute d'éducation, était le commerce de valise auquel elle s'adonnait et qui était sa principale préoccupation. Elle avait tout d'abord obtenu de l'agence qu'elle se fournisse auprès de l'épicerie de son mari pour les subsistances des pèlerins. Ensuite, elle s'est employée, dans les deux principales villes où furent effectués les achats de l'équipée, à Damas et à Gaziantep, à se procurer les biens dont on lui avait passé commande à Téhéran. Ainsi, elle s'est rendue en taxi collectif, et au prix d'une correspondance, à Bab Touma, la porte orientale de Damas, pour y trouver une chemise de nuit et une paire de chaussures de luxe dont la qualité contrastait avec les produits chinois en vente autour de Zeynabieh. Enfin, elle a acheté quelques paquets de pansements nasaux dont la mode fait fureur à Téhéran chez les jeunes filles désireuses de faire croire qu'elles ont réalisé une opération de chirurgie esthétique - les voies de la distinction sont impénétrables ! Par ailleurs, à Zeynabieh, lorsque vint l'après-midi réservé aux emplettes, le guide prit soin de préciser, avec emphase, aux tenanciers de deux magasins, l'un de cosmétiques, l'autre de chocolats, que les voyageurs étaient ceux de Haj khanoum - «Haj khanoum est le guide (moallem)» -, et donc, 
sous-entendu, que la commission devait lui être versée. Et, à notre hôtel, Haj khanoum invita un marchand de chemises et de foulards sur les ventes duquel elle toucha également sinon un pourcentage, du moins un cadeau.

Ces notations suffisent à établir que le pèlerinage arrache une femme comme Haj khanoum non seulement à son rôle traditionnel, mais encore à celui d'organisatrice de jalesseh, et même à la dimension de son quartier. Dans ce cadre elle se comporte comme l'égale d'un homme et comme une entrepreneuse opérant dans la sphère internationale. Il est particulièrement remarquable que Haj khanoum ait en l'occurrence accompagné notre groupe à Damas alors que son mari, au même moment, se rendait lui à Sareine, laissant l'épicerie à la responsabilité d'un fils : dans ce couple décidément très dynamique, l'international est laissé à l'épouse. Cette dernière possède désormais une compétence commerciale indéniable, se jouant des frontières et de la distance, comparant les produits, appréciant leur valeur, négociant leur achat, et mobilisant parents et relations pour mener à bien ses affaires.

Le cas de Haj khanoum est corroboré par celui de la femme du guide. Celle-ci nous a rejoints à Damas avec sa fille en avion, en prétextant de la longueur du voyage et de la fragilité de sa constitution. De fait sa mise et sa manière d'être se voulaient distinguées et faisaient contraste avec le style plus traditionnel de Haj khanoum et de la plupart des autres pèlerines. Même dans les sanctuaires elle ne portait pas le tchador, mais des foulards de couleur recherchés dont elle changeait deux fois par jour. Des voyageurs continuant vers Karbala et laissant des places libres dans l'autocar, la femme du guide rentra avec nous par la route. Elle prétendit modifier l'ordonnancement des places, ne cessa de parler de tout et de rien - mais surtout de ses achats - pendant tout le trajet, actionnait son compteur digital de salavat, et, s'étant emparé du téléphone portable de son mari, répondit en minaudant aux multiples appels des familles qui s'inquiétaient de la progression de l'autobus vers Téhéran.

Or, les simples pèlerines ne sont pas en reste. Elles aussi font preuve d'initiative, accumulent du savoir et de l'expérience, affrontent des situations inédites, se livrent au commerce et tirent de l'aventure un surcroît de prestige ou de distinction social une fois retournées dans leur famille et leur quartier. Une scène a été très révélatrice de cette petite révolution des habitudes et des convenances que représente un pèlerinage. Alors que nous attendions la fin d'une réparation de l'autocar sur une station-service, en Turquie, un groupe de pèlerines commença à chanter et à plaisanter. Une carriole tirée par un mulet passant sur l'aire, nous avons proposé au cocher de nous faire faire un tour, ce qu'il consentit de bon gré. Et de voir quelque sept femmes en tchador emportées par un mulet au galop, riant et chantant: une scène inimaginable dans une sociabilité urbaine en Iran. Elle provoqua naturellement la désapprobation et les grommellements de l'équipage qui néanmoins n'en pouvait mais... 
En fait, pendant toute la durée du voyage, l'on peut observer une inversion des rôles presque complète. Ce sont des hommes qui cuisinent, lavent la vaisselle, font les courses, servent tandis que les femmes, en tant que passagères, touristes et fidèles, se font servir, se distraient, s'adonnent aux joies du shopping et au vertige du commerce. De retour au pays, les femmes peuvent également briller en société, se félicitant de n'avoir rien fait d'autre que « manger et dormir pendant quinze jours ", narrant l'aventure, reconnaissant à la télévision les lieux visités, visionnant en famille les inévitables vidéos, restant en relation avec les compagnes de pèlerinage. C'est bel et bien une atmosphère de " grandes vacances » que véhicule le voyage à Damas, avec la gueule de bois ou la nostalgie du retour dans le train-train de la vie téhéranaise. Il n'empêche qu'il reste le narratif, et à lui seul celui-ci est porteur d'une transformation de la condition sociale des femmes. Non que la magie des mots suffise, mais elle s'accompagne de changements objectifs dans les rapports d'affection et de pouvoir et dans l'autonomie intellectuelle et économique des pèlerines. D'autant que ces dernières se targuent d'une qualité religieuse relevant de la transcendance : elles ont été appelées, et donc choisies, élues (talabideh shodand).

\section{Pèlerinage et structuration de la société civile}

En Iran, comme dans les autres sociétés musulmanes, la commémoration des saints est un champ de religiosité autonome par rapport à celui que contrôle le clergé. Mais le fait nouveau est que ce champ se féminise. Auparavant seules Hazrat-e Masoumeh, la sœur de l'imam Reza, et, dotée d'une moindre aura dogmatique, Bibi Shahrbanou, la fille du dernier roi Sassanide, l'épouse de l'Imam Hossein et donc la mère du Quatrième Imam chiite ${ }^{9}$, faisaient l'objet d'un culte, respectivement à Qom et dans la banlieue sud de Téhéran. Les autres saintes n'avaient de rayonnement que très local. En revanche la dévotion des pèlerins se rendant à Damas se porte en priorité, et presque exclusivement, sur une femme et une enfant : Hazrat-e Zeynab et Roghieh. Et cette vénération provient aussi bien des hommes que des femmes. L'affirmation sociale de ces dernières à travers la pratique du pèlerinage trouve donc son expression symbolique au cour même de la sacralité. Or le culte de ces saintes confirme la règle. Il échappe largement à l'emprise du clergé et de la République, ou en tout cas se situe à la marge de la religiosité officielle. Il est d'ailleurs révélateur que la mosquée de Hazrat-e Zeynab, malgré sa chiisation croissante ${ }^{10}$, soit un lieu de rencontre entre chiites et sunnites et de brouillage de la distinction confessionnelle :

9. Ali amir-Moezzi (M.), «Shahrbanu, Dame du pays d’Iran et mère des Imams : entre l'Iran préislamique et le shiisme imamite », Jérusalem Studies in Arabic and Islam, 27, 2002.

10. Mervin (S.), art. cité, p. 153-154. 
lors des grandes veillées de récitation du Coran, les sunnites (et arabes) sont sans doute très majoritaires, ce qui n'empêche nullement les pèlerins chiites (et persanophones) d'y participer.

Certes la gestion du sanctuaire de Hazrat-e Roghieh, dans le centre de Damas, est dans la main de l'Organisation iranienne de waqf qui a entièrement réaménagé le lieu et imposé l'usage du persan pour les cérémonies. En revanche Hazrat-e Zeynab est sous la coupe des autorités religieuses syriennes, même si les dons des chiites d'Irak, d'Iran et des autres États du Golfe sont très élevés, selon les autorités du sanctuaire elles-mêmes. Aussi le premier sanctuaire est-il prisé des pèlerins iraniens qui se contentent de visites plus brèves à Zeynabieh, entre deux séquences de shopping et pour les besoins du témoignage vidéo. Mais dans les deux cas le fidèle se retrouve en définitive seul avec lui-même face à son Dieu, par l'intercession des saintes. Lorsqu'ils arrivent à Zeynabieh, les pèlerins traversent rapidement la cour de la mosquée, le cas échéant sous l'œil de la caméra vidéo et sans répugner à bavarder ou à plaisanter. Une fois entrés dans le sanctuaire à proprement parler, ils se recueillent d'abord autour du mausolée en glissant à travers les grilles quelque offrande, argent ou objet personnel. Ensuite ils prient, s'abîment dans la lecture d'un livre religieux, distribuent des mets aux autres fidèles pour honorer un vœu, pleurent en se confiant. Ce qui frappe alors, c'est l'intimité du dialogue intérieur entre le croyant et son saint ou son Dieu. Les pèlerins sont quasiment serrés les uns contre les autres compte tenu de l'affluence, mais en dépit de cette promiscuité ils sont comme seuls au monde en ce moment où ils se sentent décidément appelés.

Il ne faut bien sûr pas avoir de cette religiosité une idée naïve ou culturaliste. D'une part, ces séquences sont brèves et rares, y compris à l'aune du pèlerinage. Elles ne peuvent être généralisées comme étant représentatives de la vie religieuse et encore moins sociale des chiites. D'autre part, elles n'excluent pas le marchandage ou le chantage avec Dieu ou ses saints. Au moment de quitter Téhéran le guide avait ainsi invité les voyageurs à ne réciter que la première sourate de la prière du départ et à garder la seconde pour l'arrivée sain et sauf à Zeynabieh : à charge pour la sainte de garantir la sécurité de ses orants si elle veut entendre la totalité de la prière. Et la femme du colonel, qui avait acheté comme il se doit les poupées dévotionnelles offertes à Hazrat-e Roghieh et revendues en fin de journée par le personnel du sanctuaire à de nouveaux pèlerins - les bénéfices de l'opération allant au waqf - nous expliqua qu'elle les emporterait à Téhéran et ne les rapporterait sur les lieux que si la sainte voulait bien exaucer ses demandes.

Mais l'important est cette complémentarité essentielle entre la dimension collective et la réalisation personnelle qui est à la base de l'économie morale du pèlerinage. L'individuation du croyant est portée par son appartenance à la communauté des pèlerins. Ceux-ci partagent la fatigue et les joies du voyage, sont unis dans une même sociabilité, tendent au même but, mais prient en 
solitaires. Lorsque l'une d'entre elles - le phénomène semble spécifiquement féminin pour ce que nous avons observé - manifeste une émotion particulière, voire un état de transe, dans une cérémonie, les autres fidèles l'interpellent afin qu'elle prie pour eux, mais évitent de s'interposer dans son dialogue avec Dieu.

Cette interaction entre l'engagement collectif et la logique d'individuation nous semble être un élément constitutif de la société civile iranienne dans son rapport à l'État ${ }^{11}$. Elle nourrit l'autonomie du social par rapport au champ politique et à l'institution cléricale. Le pèlerinage charrie des flux financiers considérables que ne contrôlent entièrement ni cette dernière ni le pouvoir politique. En outre il est propice à l'émergence de nouvelles manifestations de religiosité à l'initiative des fidèles eux-mêmes et, horresco referens, des croyantes ellesmêmes. La mise et le comportement des pèlerins, des pratiques de dévotion et de don comme l'offrande des poupées, les chants, le choix des livres religieux et même le zapping entre la prière et le shopping feraient pâlir plus d'un ayatollah. Enfin, nous l'avons vu, le pèlerinage est autogéré par des opérateurs privés à l'interface du commerce et du religieux, qui dans les faits ne sont pas soumis à la tutelle de la République, si tant est que l'on puisse en parler comme d'une entité homogène, centralisée ou cohérente.

Or, le pèlerinage, en tant que pratique de dévotion, n'est pas isolé et recoupe toute une série d'autres phénomènes religieux connexes, tels que les réunions de quartier du type jalesseh (pour les femmes) ou heyat (pour les hommes), le culte des morts, la célébration populaire ou juvénile d'Achoura. Dans ce dernier cas, par exemple, heyat et jalesseh se sont multipliés, qui préparent veillées et processions, ces dernières étant l'apanage des hommes et les femmes se contentant d'en être les spectatrices. Ces formes de sociabilité tendent à rassembler des personnes d'un même quartier ou d'une même profession, mais n'excluent pas une certaine hétérogénéité sociale dans la mesure où leur fréquentation reste largement familiale et où les quartiers ne sont pas eux non plus socialement homogènes : en définitive des personnes de milieux et d'âges différents s'y retrouvent. Cette multiplication des heyat et des jalesseh voués à la célébration de la mort d'Hossein est propice à toutes sortes d'innovations: les chanteurs sont désormais plus jeunes et n'ont pas nécessairement de formation cléricale; ils introduisent volontiers dans leur répertoire des poèmes de Hafez ou des évocations de l'expérience de la guerre, même s'ils ne l'ont pas euxmêmes vécue ; ils recourent naturellement aux moyens modernes de sonorisation. En outre les étendards qui sont déployés à l'occasion des veillées et des processions sont plus colorés et plus grands qu'auparavant. La préparation de ces festivités donne lieu à des effets de distinction dans le domaine vestimentaire ou dans celui de la coiffure. La réception des fidèles est plus soignée et fastueuse 
que jadis, et l'on saisit immédiatement qui est l'hôte ou l'hôtesse, saluant les fidèles à l'entrée de la cour ou de l'appartement abritant la commémoration. Comme il se doit cette évolution n'est pas du goût de toutes les vieilles barbes. Mais les jeunes comme les femmes ne manquent pas de rétorquer que l'hommage qu'ils entendent rendre à Hossein leur appartient. Il est d'ailleurs révélateur que la dénomination des heyat et des jalesseh se fait plus intime : on parlera par exemple du heyat Hossein joon, joon étant une expression familière d'affection se distinguant de la formule plus littéraire de jân (mon âme), au lieu du heyat des Deux Innocents, des Martyrs de Karbala, de Ya Aba Abdellah, etc.

Pareillement le culte des morts est l'occasion de nombreuses réunions commémoratives auxquelles assistent parents, voisins, amis et collègues et qui là aussi s'accompagnent de repas, de chants et de musiques, de transports sur les sépultures, étant entendu que des cimetières comme celui de Behesht-e Zahra à Téhéran sont de mieux en mieux aménagés pour accueillir ces manifestations. La sociabilité religieuse est ainsi une dimension privilégiée de l'initiative sociale puisque la plupart de ces mobilisations sont autogérées. Mais elle comporte également son économie politique spécifique en donnant lieu à de multiples sous-traitances dans la restauration, les performances musicales ou les déplacements, sous-traitances qui échappent tant au clergé qu'au fisc. Elle est de la sorte un lieu de prédilection de la "seconde économie » qui prospère depuis deux décennies. En définitive la problématique de la société civile qu'avait mise en avant le candidat Mohammad Khatami, en 1997, s'adressait dans les faits autant à ces dynamiques sociales, que portaient d'ores et déjà les classes moyennes et populaires, qu'aux intellectuels en mal d'espace public à la Habermas. L'espoir politique qu'il a incarné exprimait cette liberté d'entreprendre, fût-ce pour le service de Dieu et des siens, et non pas simplement le projet, au demeurant assez vague, des réformateurs qui se sont vite avérés déconnectés des préoccupations ordinaires des électeurs et se sont enlisés dans la lutte factionnelle.

De ce point de vue le pèlerinage n'a pas été la moindre de ces pratiques sociales qui ont préparé l'ouverture de la deuxième moitié des années 1990, à la fois par ce qu'il représentait en termes de mouvement et de changements sociaux et par ce qu'il véhiculait en termes d'accumulation économique informelle. Que l'on en juge. Avant de franchir la frontière turque en route pour Damas, le chauffeur et ses aides n'avaient pas manqué de charger l'autocar avec un maximum de jerricans d'essence pour les revendre en Turquie, le prix du carburant en Iran étant particulièrement bas et encourageant des exportations frauduleuses massives dans les pays avoisinants. Nous attendîmes aux environs de Malatiya, dans un jardin, une journée entière, officiellement pour nous reposer, mais en réalité - nous le découvrîmes plus tard - pour ne pas arriver trop tôt à Gaziantep où devait être revendue l'essence. Le car nous laissa alors dans la principale allée commerçante réservée aux voyageurs iraniens, Turkmen Caddesi, et disparut pendant près de quatre heures pour des raisons " techniques ». Dans cette même ville l'équipage acheta des couvertures, une par voyageur, 
mais nous dissuada d'en faire autant, arguant de leur inévitable saisie par les douaniers syriens. En réalité il utilisait notre franchise personnelle et revendit le stock à notre arrivée à l'hôtel, à Zeynabieh, où l'attendait un intermédiaire. Sur le chemin du retour le même équipage taxa chacun des cartons chargés à Gaziantep par les voyageurs - essentiellement de l'électroménager - et l'une des pèlerines, qui en avait dix-huit à elle seule, n'hésita pas à attribuer l'origine de l'accident dont nous fûmes victimes à la rapacité du conducteur. Si l'on ajoute à ce commerce le prix des billets, la vente des enregistrements vidéo réalisés par le caméraman, les diverses commissions que rétrocèdent au guide ou à Haj khanoum les restaurants, les hôtels et les négoces fréquentés par les pèlerins, et enfin les oboles recueillies à plusieurs reprises pendant le voyage dans un sac en plastic, l'on voit qu'un pèlerinage représente bien un flux financier conséquent.

En se dérobant en grande partie à l'État sans pour autant le remettre en cause, ce dernier participe de l'autonomie relative du social qui concourt à la structuration d'une société civile, en l'occurrence religieuse dans son économie morale. Un autre paradoxe vient alors de ce que ce flux financier ou économique informel, et les dynamiques sociales transnationales qui en sont le support, enregistrent et nourrissent en définitive la conscience nationale par le biais de cette économie morale et religieuse, bien que celle-ci s'affirme universelle, en se référant à l'ummat, ou en tout cas sans frontières, en se définissant par rapport à la mouvance chiite régionale qui s'étend peu ou prou de l'Inde au Levant en passant par le Golfe.

\section{Une économie politique et morale transnationale au service de la conscience nationale}

Il est inutile de rappeler longuement que l'ummat est un principe d'universalité, et que la communauté des chiites est elle-même transnationale dans son organisation cléricale et dans sa circulation régionale des fidèles, d'un lieu de pèlerinage et de commerce à un autre. Bien sûr, le débat politique contemporain tend à occulter ces vérités premières en mettant l'accent sur l'identité islamique ou confessionnelle et sur les conflits qu'elle suscite. De l'islam on ne voit plus que l'islamisme, voire le terrorisme, et du chiisme que les affrontements intercommunautaires dont il est partie prenante, par exemple au Liban, dans les monarchies du Golfe et au Pakistan. Mais, outre le fait que la foi musulmane est l'une des grandes religions qualifiées d'universalistes, l'enseignement de la théologie chiite, la perception des impôts religieux par les clercs et leur périmètre d'autorité, l'organisation des waqf et des réseaux financiers (hawala, banques islamiques) dont ils sont les tenants, le droit islamique lui-même, sont résolument transnationaux. A cette aune des villes saintes comme Nadjaf et Karbala sont en quelque sorte off shore, et de ce point de vue la chute de Saddam Hussein devrait leur rendre tout leur lustre. De même cursus théologiques et alliances matrimoniales au 
sein du clergé chiite compliquent les appartenances nationales des clercs : grande figure du chiisme libanais, Moussa Sadr était d'origine iranienne et apparenté aux familles de l'imam Khomeiny et de Mohammad Khatami. Il n'en reste pas moins que, dans le même temps, la conscience nationale iranienne, pour environ $80 \%$ de la population, est plus ou moins indissociable de l'appartenance au chiisme, les dirigeants de la République islamique n'étant pas les derniers à insister sur cette complémentarité entre les deux identifications. Les pèlerins iraniens qui se rendent à Zeynabieh, ou aussi bien dans les villes saintes d'Irak et à La Mecque, sont au cœur de cette tension entre la coloration confessionnelle de leur nation et l'universalité de leur foi ou son inscription régionale privilégiée.

Par ailleurs nos voyageurs se voient en être-en-société (âdam-e ejtemâi), parcourant le monde, économiquement entreprenants, se livrant aux délices de la consommation, soucieux de leurs droits et de leur respectabilité, explorant de nouveaux rapports sociaux, mettant en correspondance leur vie privée et leur participation à l'espace public. Sous couvert d'une dévotion confessionnelle ils s'ouvrent au grand vent de la globalisation et accèdent également par ce biais à une certaine forme d'universalité, peinte aux couleurs de la modernité et de la mondialisation.

La géographie morale des pèlerins permet de mieux comprendre comment ces fausses contradictions sont dépassées dans les faits. Nombre d'entre eux ne se rendent pas «en Syrie », mais sur la tombe de Hazrat-e Zeynab. « Nous arrivons à Damas", avions-nous répondu à une jeune femme d'une trentaine d'années qui nous interrogeait pour savoir où nous étions. Elle ne comprit pas vraiment notre réponse, et nous eûmes à préciser: "Nous arrivons à Zeynabieh ", ce qui lui fut immédiatement intelligible. Dans la bulle de leur autocar les pèlerins parcourent un espace ô combien transnational qui est circonscrit par des lieux sacrés transcendant la géographie nationale. Durant la guerre avec l'Irak, un téléspectateur nous avait ainsi demandé si l'Euphrate dont la presse faisait quotidiennement état pour rendre compte du déroulement du conflit était bien celui au bord duquel l'imam Hossein était mort assoiffé. Pour autant le pèlerin ne se perd pas dans cette géographie morale transnationale. Il communie à elle en tant qu'individu, certes porté par une communauté circonstancielle, celle des autres voyageurs, mais c'est pour revenir en Iran, son lieu de résidence, celui de sa famille, sa patrie. Il ne cesse au demeurant de rester en contact avec les siens par le téléphone et par les achats qui leur sont destinés. Quant au commerce de valise ou de contrebande, il a bien pour marché Téhéran ou l'Iran. La nostalgie du dernier jour, qui s'exprime si fortement lors de l'ultime visite à Hazrat-e Roghieh, le sentiment que la fête est finie et que la loi médiocre de la quotidienneté va à nouveau l'emporter ${ }^{12}$, la

12. Cf. le travail de A. Madoeuf sur le temps révolu du pèlerinage, «Éphémérides de la ville en fête : une lecture des mouleds au Caire ", in Chiffoleau (S.), Madoeuf (A.), dir., Les pèlerinages au Maghreb et au Moyen-Orient, op. cit. 
joie aussi et la fierté de retrouver parents et amis auréolé de cette aventure marquent bien cette réinsertion dans l'espace national. Le pèlerinage est un départ qui ne vaut que par un tel retour. Le champ transnational ne dissout pas la dimension nationale, il la valorise et la sublime en l'enrichissant de nouvelles références et de nouvelles expériences. Le pèlerinage d'ailleurs n'est pas qu'un moment fugace, une simple parenthèse, puisque les voyageurs, revenus au pays, gardent généralement des liens entre eux, parfois intenses et émotionnels, qui contribuent à la diversification de la société civile nationale.

En outre le caractère transnational de la géographie morale du pèlerinage est tempéré par la géographie commerciale de ce dernier. Les flux de voyageurs sont tels, entre Téhéran et Damas, que tout au long du parcours les voyageurs évoluent dans un monde qui reste familier. De nombreux Iraniens se sont installés dans les principales étapes du périple et y tiennent des commerces. Quant aux gens du cru, Turcs ou Syriens, ils ont appris des rudiments de persan pour la nécessité de leurs affaires. Prestataires de services et marchands apposent des enseignes en farsi et acceptent les rials. Pareillement les pèlerins ne cessent de manger iranien et retrouvent dans les hôtels de Zeynabieh les portraits de l'imam Khomeiny et du Guide de la Révolution. Dans le cocon de cet Iran transplanté au Levant, les Iraniens peuvent ainsi mesurer leur différence par rapport aux autres pèlerins originaires du sous-continent indien, de l'Asie de Sud-Est ou du Golfe et par rapport aux Syriens eux-mêmes. Un soir deux femmes s'amusèrent à imiter une servante et une maîtresse de maison arabes, avec force pantomime et dans un fou rire généralisé : elles s'étaient outrancièrement couvert le visage de leur voile, se montraient inactives et corpulentes et gardaient le silence. Même en Terre sainte l'Arabe est l'objet de plaisanteries de plus ou moins bon goût. Et en l'occurrence la satire permettait de réaffirmer la supériorité de l'Iran dont les femmes participent à l'espace public et sont entreprenantes, elle rappelait aussi qu'il y a toujours plus soumis que soi.

Le pèlerinage fournit par ailleurs l'opportunité d'inscrire cette conscience nationale dans les processus de régionalisation du Moyen-Orient et de globalisation. Les Iraniens qui vont à Damas rencontrent des compatriotes expatriés de plus ou moins longue date qui s'adonnent aux affaires et ont une expérience de l'international sans être moins Iraniens pour autant. Ils en tirent une certaine familiarité avec le monde moderne extérieur, et même une certaine fierté : après tout les Iraniens peuvent eux aussi réussir à l'étranger, et ce sentiment conforte de manière diffuse le désir de départ qui parcourt la société ${ }^{13}$. De plus les pèlerins élargissent le périmètre national. Même s'ils ne se rendent pas toujours compte qu'ils ont affaire à des moaved, ils s'entendent désigner par le 
guide d'autres voyageurs comme étant «Iraniens » alors qu'ils possèdent la nationalité koweiti, bahreini ou émirati. Au même titre que le pèlerinage commercial à Dubaï, le pèlerinage sur les tombes de Hazrat-e Zeynab et Roghieh rend tangible la réalité sociale et historique de la diaspora qui a essaimé depuis des siècles, et plus précisément depuis le début du XX siècle, dans le Golfe, en Turquie, en Arabie et au Levant. Non seulement il ne contredit pas le sentiment national, mais il le complexifie, l'enrichit et le réactualise.

Sur le chemin du retour, trois heures avant d'arriver à Téhéran, un professeur du secondaire qui voyageait seul et avait sympathisé avec l'équipage céda à notre demande et accepta de chanter. Ayant saisi le micro du guide il entonna Morgh-e Sahar (L'Oiseau de l'aube), un poème de Mohammad Taghi Bahar qui a été adopté comme hymne par la mouvance nationaliste et dont la connotation est restée associée à celle-ci et à la résistance de la société (mellat) face à l'État $(\text { dolat })^{14}$. À aucun moment nous n'avions parlé politique durant le voyage, et il serait sans doute erroné d'accorder à ce moment de grande émotion - l'ensemble de l'autocar avait repris le chant en frappant des mains - une signification politique explicite. Néanmoins il est remarquable qu'une pérégrination transnationale s'achève sur un chœur national. Était ainsi exprimée l'unité de la nation iranienne, au-delà des clivages factionnels ou des particularismes ethnorégionaux. Et plus encore peut-être l'aspiration, à la fois nationale et personnelle, de prendre en main son destin : «ô oiseau affolé, échappe-toi de ta cage et entonne avec l'humanité le fredonnement de la liberté !». Pratique religieuse de célébration des saints, le pèlerinage, loin d'être le simple opium du peuple, s'avère être un vecteur d'éveil et de mobilisation sociaux.

\section{Conclusion}

Reste à savoir quelles sont les grandes lignes de cette économie morale de l'État et de la nation que véhicule le pèlerinage transnational. Il n'est pas question de postuler que celui-ci révèle une «culture iranienne » ou « chiite » cohérente. Mais il est porteur de quelques valeurs éthiques et de quelques pratiques qui semblent indissociables de l'expérience historique de la société iranienne en République islamique. La première évidence a trait à l'autonomisation sociale des femmes : c'est un phénomène absolument inédit que de les voir voyager, développer des formes cultuelles spécifiques et commercer indépendamment de leur mari, et ce à l'étranger aussi bien qu'à l'intérieur du pays. Un autre trait marquant est l'individuation des pèlerins, quel que soit leur sexe ou leur âge. Ces derniers voyagent d'abord en tant qu'individus, en laissant derrière eux

14. Katouzian (H.), « The Short-Term Society : a Study in the Problems of Long-Term Political and Economic Development in Iran », Middle Eastern Studies, 40 (1), 2004. 
l'entité de la famille, ce qui naturellement n'exclut ni le fait qu'ils peuvent être éventuellement accompagnés par un membre de celle-ci, ni le fait qu'ils restent en contact permanent avec ceux qui sont restés au pays par le biais du téléphone ou de l'émotion. En troisième lieu l'expérience religieuse, tout en gardant sa transcendance et son irréductibilité, va de pair avec l'omniprésence du calcul économique rationnel: la sacralité et les affaires sont concomitantes sans se confondre, et le pèlerin, tout à sa dévotion, consacre une part déterminante de son déplacement à la mise en œuvre d'une stratégie commerciale. En quatrième lieu, le pèlerinage confronte les voyageurs aux inconnues de l'étranger et introduit un élément de relativisation et d'autoréflexivité de la conscience nationale : le dépaysement géographique et linguistique, la séparation d'avec les proches, les imprévus de la route représentent un changement d'échelle sociale et introduisent là aussi une rupture avec les usages antérieurs. D’autant que l'expérience de la pérégrination religieuse s'adosse à celle, croissante, de l'expatriation économique et s'insère dans les circuits de la diaspora. Enfin, si le voyage continue d'être une ressource de connaissances, de sagesse et de distinction, plus encore aujourd'hui que dans les anciens temps, il se trouve légitimé par le but religieux du pèlerinage qui en quelque sorte «blanchit» les intentions de celui qui part et qui pourrait toujours être soupçonné de se livrer à des activités économiques ou à des plaisirs condamnables.

Fariba ADELKHAH est anthropologue, directrice de recherche à la Fondation nationale des sciences politiques (Centre d'études et de recherches internationales, CERI). Spécialiste de I'Iran, elle s'intéresse en particulier à la place du religieux dans la centralisation du pouvoir et la "modernisation " des comportements politiques. Ses recherches actuelles portent sur l'anthropologie du voyage et l'économie informelle comme mutation des régulations de l'État et des relations entre la société iranienne et son environnement régional et international.
Elle a notamment publié : La Révolution sous le voile. Femmes islamiques d'Iran, Paris, Karthala, 1991, L'Iran, Paris, Le Cavalier bleu, 2005 et Être moderne en Iran, Paris, Karthala, 2006 (nouvelle édition augmentée) ; "Les Afghans iraniens ", en collaboration avec Zuzanna Olszewska, Les Études du CERI, n 125, avril 2006 (disponible sur le site www.ceri-sciencespo.com). 